\title{
Tracker Alignment Strategy in CMS and Experience with Cosmic Ray Data
}

\author{
Gero Flucke* \\ (on behalf of the CMS collaboration) \\ University of Hamburg, Luruper Chaussee 149, 22761 Hamburg, Germany \\ E-mail: gero.flucke@cern.ch
}

\begin{abstract}
Aligning the CMS silicon tracker is an unprecedented challenge due to the more than 15000 modules that it is composed of. Besides high precision mounting, survey measurements and the laser alignment system, track based alignment is needed to reach the envisaged precision. The first successful full scale alignment study on simulated data uses a fast global minimisation procedure implemented in the Millepede II program. Tracks from cosmic ray muons and the inclusion of prior knowledge are key ingredients. Experience beyond simulation studies is gained analysing real data from cosmic ray muons.
\end{abstract}

The 16th International Workshop on Vertex detectors September 23-28 2007

Lake Placid, NY, USA

${ }^{*}$ Speaker. 


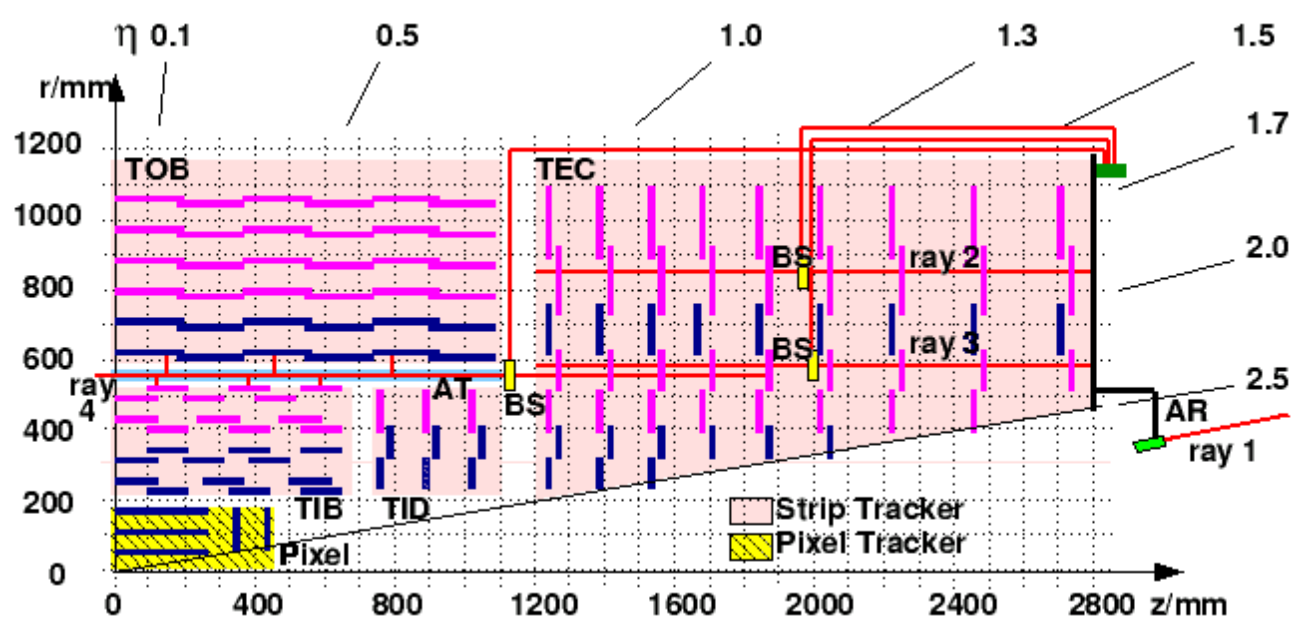

Figure 1: Cross section through a quarter of the CMS Tracker in $r z$-view, showing Strip and Pixel detector together with the Laser system. The blue lines indicate two strip modules mounted on top of each other with a stereo angle to allow 2D measurements.

\section{Introduction}

The CMS Tracker [1] comprises both a silicon pixel and a silicon strip detector within a diameter larger than $2 \mathrm{~m}$ and a length larger than $5 \mathrm{~m}$, as shown in Fig. 1 and summarised in [2]. The Pixel Tracker is built from 1440 pixel modules with a pixel size of $100 \mu \mathrm{m}(150 \mu \mathrm{m})$ in $r \phi(z)$ direction. It consists of a barrel (BPIX) and a forward (FPIX) part. A single hit resolution of up to $9 \mu \mathrm{m}(20 \mu \mathrm{m})$ is achieved. The Strip Tracker is subdivided in mechanically separate parts: Inner Barrel (TIB), Outer Barrel (TOB), Inner Disk (TID) and Endcap (TEC). All parts together comprise 15148 single-sided silicon strip modules with strip pitches ranging from $80 \mu \mathrm{m}$ to $205 \mu \mathrm{m}$. The single-strip resolution varies from $23 \mu \mathrm{m}$ to $60 \mu \mathrm{m}$ in the sensitive coordinate. For each possible track trajectory, at least four measurements are obtained in the strip tracker by a pair of modules assembled back-to-back with a stereo angle of $100 \mathrm{mrad}$, leading to a stereo resolution of $230 \mu \mathrm{m}$ to $530 \mu \mathrm{m}$.

In order not to deteriorate the track parameter resolutions significantly, the positions of the modules have to be known to a precision below $10 \mu \mathrm{m}$ for their sensitive coordinates. Depending on the module size, this corresponds to an angular precision of $100 \mu \mathrm{rad}$. This is beyond the mounting accuracy achieved. To reach the desired precision, a dedicated alignment strategy has been employed to determine the position and orientation of all the 16588 silicon modules. This strategy includes precise assembly, survey measurements (Sec. 2), the laser alignment system (LAS, Sec. 3) and track based alignment (Sec. 4). Figure 2 shows how this is estimated to improve the physics performance with increasing available data sets and experience. First real data experience is currently gained with analysing cosmic ray data (Sec. 5).

\section{Precision Assembly and Survey}

During construction and assembly of the CMS Tracker, a vast number of measurements have been performed, e.g. by coordinate measurement machines or photogrammetry, to verify the de- 

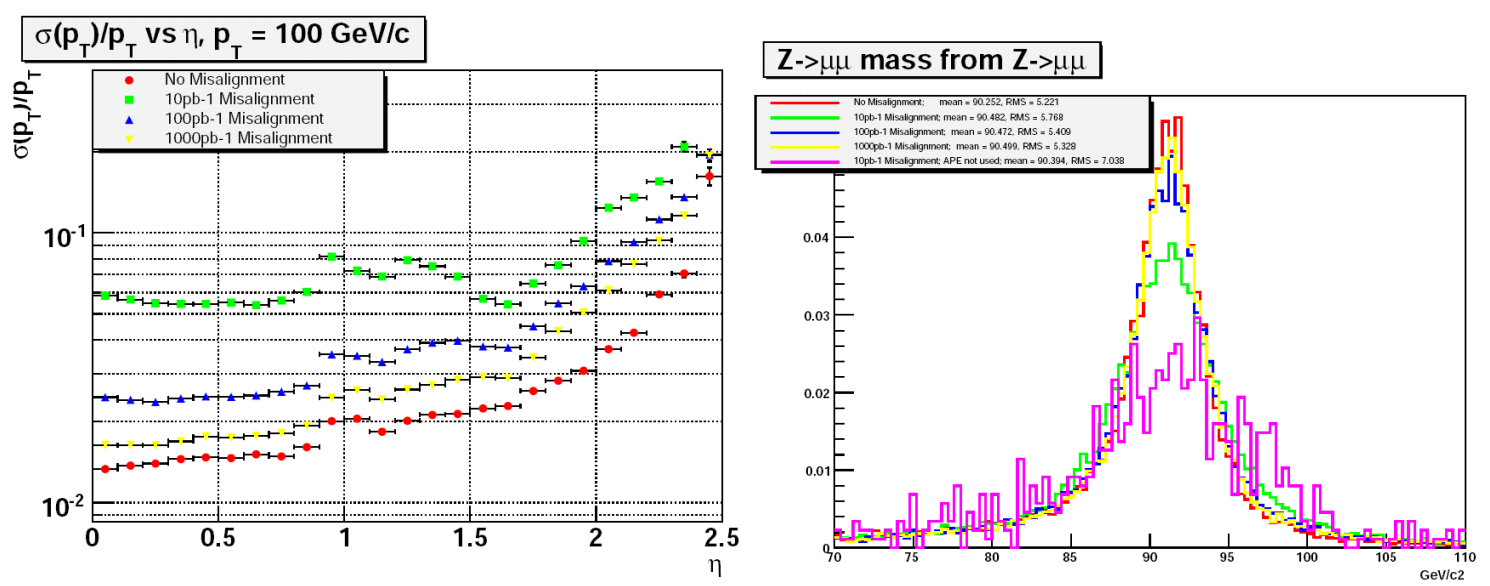

Figure 2: Estimated improvement of the performance of the track measurements using refined alignment constants expected after accumulating data from an integrated luminosity of 10 (green), 100 (blue) and $1000 \mathrm{pb}^{-1}$ (yellow) compared to ideal alignment precision (red): Transverse momentum $\left(p_{t}\right)$ resolution for single muons with $p_{t}=100 \mathrm{GeV} / c$ as a function of the pseudorapidity (left) and reconstructed mass peak for $Z^{0} \rightarrow \mu^{+} \mu^{-}$decays (right).

\begin{tabular}{|l|r|l|l|l|l|l|l|}
\hline \multicolumn{2}{|c|}{ TIB } & \multicolumn{2}{|c|}{ TID } & \multicolumn{2}{c|}{ TOB $(r \phi / z)$} & \multicolumn{2}{c|}{ TEC } \\
\hline Sensor & 10 & Sensor & 10 & Sensor & 10 & Sensor & 10 \\
Module & 180 & Module & 54 & Module & 30 & Module & 20 \\
Shell & 450 & Ring & 54 & Rod & 185 & Petal & 70 \\
Cylinder & 750 & Disc & 350 & Wheel & 100 & Disc & 150 \\
Tube & & Cylinder & 450 & Tube & 1000 & TEC & 600 \\
& & Tube & & CMS & & Tube & \\
\hline
\end{tabular}

Table 1: Estimated assembly precision (RMS, in $\mu \mathrm{m}$ ) of strip tracker components [1]. Values are given in between the mechanical hierarchy levels they are valid for, e.g. the position accuracy of sensors in modules is $10 \mu \mathrm{m}$.

sired mechanical accuracy for most Tracker components. Table 1 summarises the estimated assembly precision in the strip detector between the different hierarchy levels of the mechanical structures.

For the TIB, TID and FPIX detectors a very detailed survey of all structural levels down to module positions has been performed. For TOB and TEC the smaller structures have been measured only on a sample basis. For the BPIX a partial survey is planned, limited by time constraints.

\section{The Laser Alignment System}

The Laser Alignment System (LAS) [1] uses infrared laser beams with a wavelength $\lambda=$ $1075 \mathrm{~nm}$ to monitor the positions of selected tracker modules. In an eightfold $\phi$-symmetry, there are laser beam lines through the nine disks of each TEC at two different radii and beam lines connecting the TECs with the TIB and TOB only. The beam positions are directly detected on silicon sensors 

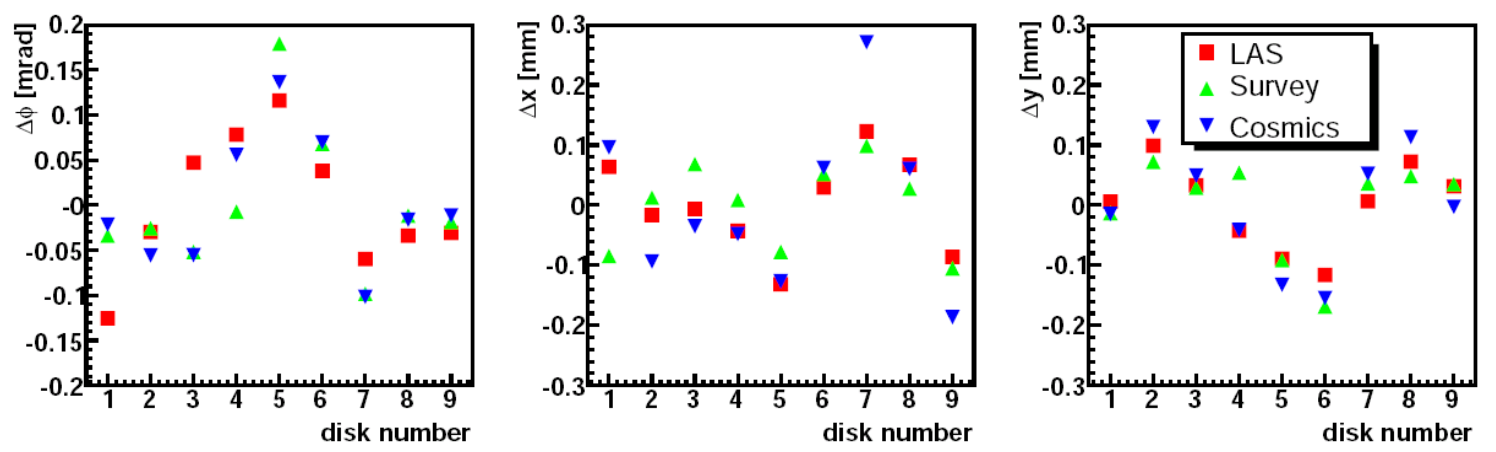

Figure 3: TEC $(z>0)$ disk rotation $\Delta \phi$ (around the beam axis) and displacements $\Delta x, \Delta y$ (in the disk plane) as determined from survey, LAS and cosmic muon tracks.

also used for track reconstruction, though they are specially treated to allow transmission of the laser light. Figure 1 shows which modules are connected by the different laser beam lines.

The goal of the system is to generate alignment information on a continuous basis, providing geometry reconstruction of the tracker substructures at the level of $100 \mu \mathrm{m}$, which is mandatory for High Level Trigger (HLT) of CMS. In addition, possible tracker structure movements can be monitored at the level of $10 \mu \mathrm{m}$, providing additional input for the track based alignment.

During integration of the TEC at $z>0$, deviations of disk positions and orientations from nominal values have been determined from survey with photogrammetry, the LAS, and tracks from cosmic muons. Figure 3 shows the results from the three complementary methods. The global degrees of freedom (absolute position and orientation, torsion and shear around the symmetry axis) have been fixed to be zero. The deviations from ideal geometry are of the order of $\mathscr{O}(100 \mu \mathrm{m})$ and $\mathscr{O}(100 \mu \mathrm{rad})$, respectively, and agree well with each other, setting an upper value on the precision of each method.

\section{Track Based Alignment}

The final tool to achieve the desired alignment precision is track based alignment. The common principle is the minimisation of an objective function ${ }^{1}$ built up from the normalised unbiased track hit residuals. CMS has employed or interfaced three different algorithms:

HIP The Hits and Impact Point algorithm [3] minimises the sum of the residuals of each aligned object, independently from the others. Since the track fit predicting the track impact point is biased by misalignment, this is repeated iteratively until convergence is assumed.

Kalman This algorithm is an extension of the Kalman filter used in track fitting by including alignment parameters. These are updated after each processed track. The computational complexity is reduced by restricting the update to detectors that are close in the sense of a certain metrics [4].

Millepede II is an upgraded version of the Millepede program [5]. The basic principle is the minimisation of the linearised objective function, simultaneously taking into account track and

\footnotetext{
${ }^{1}$ In high energy physics usually called $\chi^{2}$-function.
} 
a)

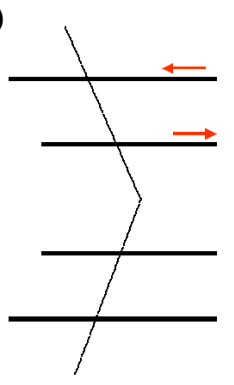

b)

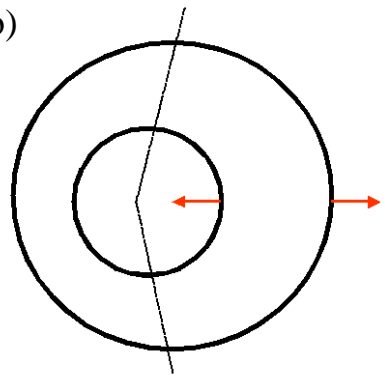

c)

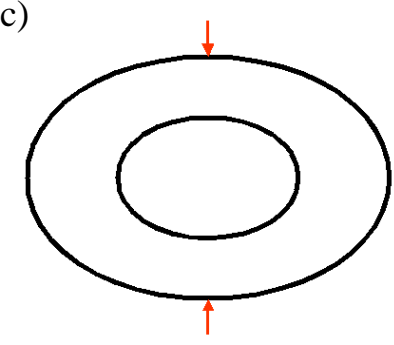

d)

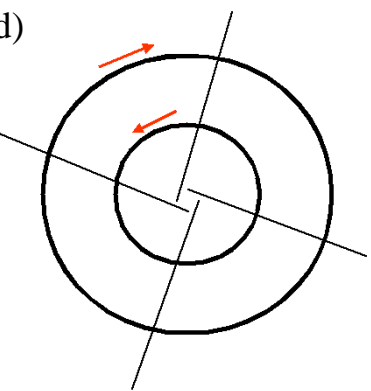

Figure 4: Illustration of deformations that hardly influence the objective function value to be minimised in track based alignment if only single tracks from the interaction point are used.

alignment parameters. Being interested only in the $n$ alignment parameters, the problem is reduced to the solution of a matrix equation of size $n$ without information loss. Millepede II [6] offers fast methods to solve this equation for up to $n=100000$, sparse matrix storage, hit outlier rejection and downweighting, constraints to fix e.g. the global degrees of freedom, the inclusion of e.g. survey measurements, and assignment of a priori uncertainties to parameters.

Since embedded in the CMS Alignment Framework, these algorithms can switch to align single modules or larger objects, following the mechanical structures. Thus they are all easily adjustable to available statistics.

A major challenge for track based alignment are the so called weak modes, i.e. deformations of the geometry that have no (or weak) influence on the value of the objective function, but may bias the measurements. They are intrinsic to the problem and independent of the algorithm, but they depend on the data sample used. For example, the weak modes illustrated in Fig. 4 are suppressed by using cosmic tracks that connect opposite detector parts. CMS foresees to utilise the following data sets:

Muons from $Z^{0}$ and $W$ decays used as single tracks will be abundant at high luminosity and show only little multiple scattering.

Cosmic Ray Muons relate opposite detector parts and are thus essential to reduce weak modes.

Beam Halo Muons from beam induced background are more or less parallel to the beam and therefore compensate for the low cosmic ray statistics in the endcaps.

Mass Constrained Muon Pairs from $Z^{0} \rightarrow \mu^{+} \mu^{-}$and $J / \Psi \rightarrow \mu^{+} \mu^{-}$set a momentum scale. A vertex constraint is included.

Minimum Bias Tracks with a minimum momentum are abundant already in the beginning.

Vertex Constrained Tracks, even from minimum bias, suppress e.g. the weak mode in Fig. 4d.

LAS provides straight track like measurements in the endcaps.

\subsection{Full Tracker Alignment Study}

A study on simulated data demonstrates that the Millepede II algorithm is able to align the full CMS tracker [7]. Starting with a pixel tracker pre-aligned to about $15 \mu \mathrm{m}$, it utilises single muon 

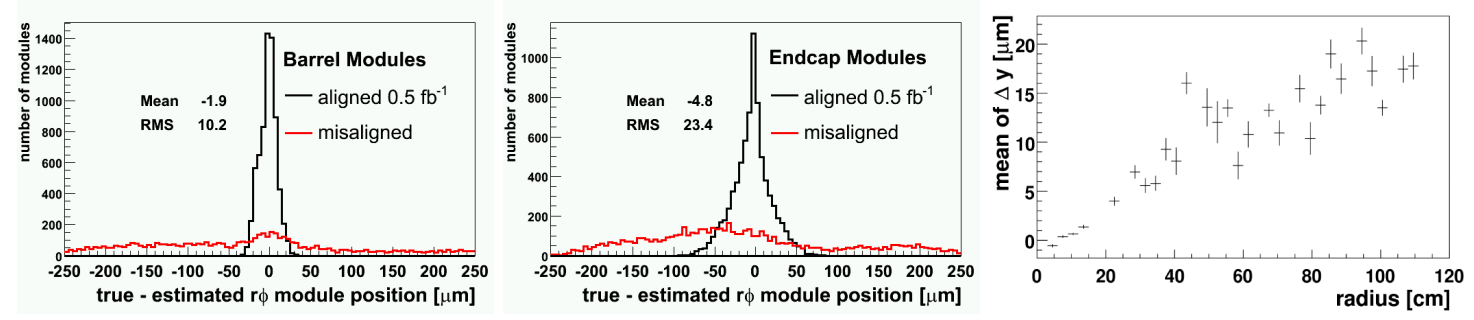

Figure 5: Remaining misalignment of the precisely measured coordinate for barrel (left) and endcap (middle) modules, and mean vertical shift as a function of the radius (right).

tracks and mass/vertex constrained pairs of muon tracks from $W^{ \pm}$and $Z^{0}$ decays, respectively, corresponding to an integrated luminosity of $L=0.5 \mathrm{fb}^{-1}$. As a very important dataset, high momentum cosmic muon tracks of a few weeks of dedicated data taking are used in addition. Studies show that it is possible to achieve about $10 \mathrm{~Hz}$ of cosmic ray tracks exceeding $p=5 \mathrm{GeV} / c$ in the tracker volume. The alignment parameters taken into account for all modules are a shift along the precisely measured coordinate (close to parallel to the $r \phi$-direction), a shift perpendicular to the sensor plane and a rotation around the sensor normal. In addition, the shift along the coarsely measured coordinate is aligned for the pixel modules and for the stereo modules in the strip detector. This amounts to about 45000 free parameters. A hierarchical parameterisation and a priori knowledge are used to suppress weak mode deformations.

The achieved precision of the resulting module positions in the precisely measured coordinate is excellent, as can be seen in Fig. 5a-b. It reaches $\sigma_{r \phi}=10 \mu \mathrm{m}$ in the barrel and even $\sigma_{r \phi}=1.2 \mu \mathrm{m}$ in the pixel barrel that defines the coordinate system. Due to missing cosmic ray tracks in the endcaps (TID and TEC), their modules reach $\sigma_{r \phi}=23 \mu \mathrm{m}$. A systematic misplacement along the global vertical $(y-)$ axis, $\langle\Delta y\rangle \propto r$, is the main source of misalignment as shown in Fig. $5 \mathrm{c}$ and can be attributed to the fact that there are no horizontal cosmic muons to fix this weak mode.

Besides the achieved precision also the modest computing needs are remarkable; the solution of the final matrix equation took less than 2 hours on a $3 \mathrm{GHz} 64$ bit PC, requiring no more than about 2 GB of memory.

\subsection{Pixel Monitoring}

The pixel detector is not included in the LAS, but fast feedback about movements is important for the HLT. Therefore the pixel detector will have to be monitored with tracks. A feasibility study shows that minimum bias tracks from about one hour of data taking at nominal LHC luminosity are sufficient to notice and correct correlated misalignment in the pixel barrel. The six pixel half barrel structures, i.e. cylinders cut through their symmetry axes that coincide with the beam line, are shifted by up to $100 \mu \mathrm{m}$ in the coordinates perpendicular to the beam axis and rotated by up to $60 \mathrm{mrad}$ in all three angles. Figure 6 shows how the HIP algorithm converges within 50 iterations to a precision better than $10 \mu \mathrm{m}$ for the shifts. The orientations are recovered to better than $100 \mu \mathrm{rad}$. Including a vertex constraint proved to be very useful. 

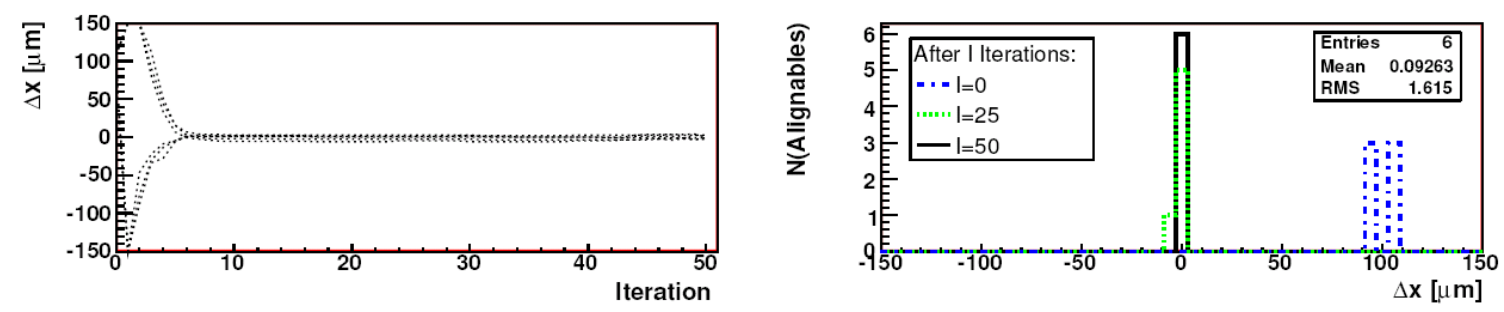

Figure 6: Convergence of one of the pixel half barrel coordinates perpendicular to the beam line. The residuals are shown as a function of the iteration on the left and projected for the initial misalignment (iteration 0) and after 25 and 50 iterations on the right.
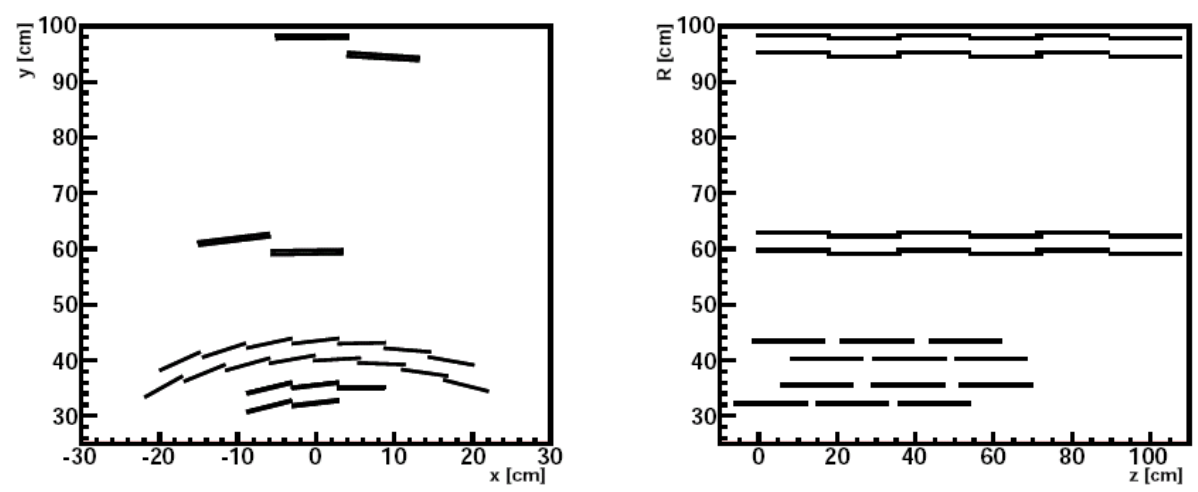

Figure 7: Custom-built sub-structure of the silicon strip tracker during the MTCC containing modules of TOB layers 1 and 5 and of TIB layers 2 and 3 in $x y$-plane (left) and in rz-plane (right).

\begin{tabular}{|c|c|c|cc|cc|}
\hline \multicolumn{3}{|c|}{} & \multicolumn{2}{|c|}{ res. TIB (RMS) $[\mu \mathrm{m}]$} & \multicolumn{2}{l|}{ res. TOB (RMS) $[\mu \mathrm{m}]$} \\
\hline & N(tracks) & $\left\langle N_{\text {hit }}\right\rangle$ & layer 2 & layer 3 & layer 1 & layer 5 \\
\hline Before & 1460 & 3.3 & 526 & 416 & 2660 & 1986 \\
\hline After & 4956 & 4.3 & 177 & 125 & 357 & 687 \\
\hline
\end{tabular}

Table 2: Number of reconstructed tracks, their mean number of hits and the RMS of the hit residuals in the four layers of the MTCC tracker setup, before and after alignment.

\section{Cosmic Ray Data}

A custom-built substructure of the strip tracker, illustrated in Fig. 7 and representing about $1 \%$ of the tracker read out channels, has taken part in the CMS Magnet Test Cosmic Challenge (MTCC) during the summer 2006. Data has been taken with a magnetic field of up to $B=4 \mathrm{~T}$. Though the largest data sample with 5962 events with hits in at least 3 different layers has been taken at $B=0 \mathrm{~T}$. Track based alignment is exercised with the HIP algorithm on that sample [8]. Due to limited statistics, the algorithm aligned larger mechanical structures called rod (in TOB) and string (in TIB), containing 6 and 3 modules, respectively. First the TOB rods have been aligned, keeping the TIB strings as reference, and then vice versa. Large alignment corrections have been found and are verified by the huge increase of the number of reconstructed tracks and the decrease of the track-hit residuals, both shown in Tab. 2.

Currently more experience is gained analysing the data taken in the Tracker Integration Facility 
(TIF) until summer 2007 with a larger subset of the strip tracker in its final configuration and readout. Tracker commissioning and track reconstruction at the TIF are presented elsewhere in these proceedings $[9,10]$.

\section{Conclusion}

The size of the CMS silicon tracker and the high position resolution of its 16588 modules poses a great challenge for alignment. This is faced by an alignment strategy based on precise assembly, many survey measurements, the laser alignment system (LAS) and track based alignment algorithms.

A part of the LAS has been successfully operated during TEC integration and confirmed the high mounting precision. During LHC operation it will provide fast feedback about movements of the strip subdetectors TIB, TOB and TEC.

As a proof of concept, an alignment study on simulated data using the Millepede II algorithm shows that the precision envisaged for track based alignment can indeed be reached for the full tracker. Besides high $p_{t}$ muon tracks and mass and vertex constrained muon pairs from $Z^{0}$ decays, cosmic ray muons and the use of a priori knowledge are essential inputs to avoid weak modes that bias the measurements. Tracks from beam halo muons and measurements of the LAS are expected to improve the results in the endcaps.

It has also been shown that the position and orientation of the pixel barrel detector can be reasonably well monitored with minimum bias tracks.

While experience with real data is currently gained analysing cosmic muon tracks recorded in the Tracker Integration Facility, we are confident to provide alignment constants applicable for the first physics analyses, monitor their validity with time and increase their precision with time according to physics' needs.

\section{Acknowledgements}

I would like to thank the CMS tracker alignment group for the possibility to present these promising results. The conference committee may take credit for the excellent organisation and the inspiring atmosphere.

\section{References}

[1] CMS-Collaboration, "The CMS Experiment at the CERN LHC", to be submitted to the Journal of Instrumentation.

[2] M. Weber, "CMS Tracker Alignment Strategy" in proceedings of 15th International Workshop on Vertex detectors, Perugia, Italy, September 25-29 2006, Nucl. Instrum. Meth. A 582 (2007) 795, [CMS CR-2007/002].

[3] V. Karimäki, T. Lampén, F.-P. Schilling, "The HIP Algorithm for Track Based Alignment and its Application to the CMS Pixel Detector", [CMS NOTE-2006/018].

[4] E. Widl, R. Frühwirth and W. Adam, "A Kalman Filter for Track-based Alignment”, [CMS NOTE-2006/022]. 
[5] V. Blobel and C. Kleinwort, "A New Method for High-Precision Alignment of Track Detectors", contribution to the Conference on Advanced Statistical Techniques in Particle Physics, Durham, England, March 18-22 2002, [hep-ex/0208021].

[6] V. Blobel, "Software Alignment for Tracking Detectors," in proceedings of Workshop on Tracking in high Multiplicity Environments, Zürich, Switzerland, October 3-7 2005, Nucl. Instrum. Meth. A 566 (2006) 5. A program description for Millepede II and the code is available via: http: //www.desy.de/ blobel

[7] M. Stoye, "Calibration and Alignment of the CMS Silicon Tracking Detector", Ph.D. thesis, Universität Hamburg (2007), [CMS TS-2007/017 and CERN-THESIS-2007-049].

[8] D. Benedetti et al., "Tracking and Alignment with the Silicon Strip Tracker at the CMS Magnet Test Cosmic Challenge", CMS NOTE-2007/030.

[9] C. Delaere et al., "CMS Tracker Commissioning and First Operation Experience" in proceedings of 16th International Workshop on Vertex detectors, Lake Placid, NY, USA, September 24-28 2007 , PoS (Vertex 2007) 002 .

[10] C. Noeding, "Track Reconstruction and Experience with Cosmic Ray Data in CMS" in proceedings of 16th International Workshop on Vertex detectors, Lake Placid, NY, USA, September 24-28 2007, PoS (Vertex 2007) 026. 